\title{
Genome-wide SNP identification in Fraxinus linking genetic characteristics to tolerance of Agrilus planipennis
}

\author{
Cecelia E. Hale ${ }^{1}$ | Mark A. Jordan ${ }^{1}$ | Gloria Iriarte ${ }^{2}$ | Kirk Broders ${ }^{3}$ | Andrew J. Storer ${ }^{4}$ | \\ Vamsi J. Nalam ${ }^{1,2}$ | Jordan M. Marshall ${ }^{1}$ (D)
}

${ }^{1}$ Department of Biology, Purdue University Fort Wayne, Fort Wayne, Indiana

${ }^{2}$ Bioagricultural Sciences and Pest Management, Colorado State University, Fort Collins, Colorado

${ }^{3}$ Smithsonian Tropical Research Institute, Panama City, Panama

${ }^{4}$ College of Forest Resources and Environmental Science, Michigan Technological University, Houghton, Michigan

\section{Correspondence}

Jordan M. Marshall, Department of Biology, Purdue University Fort Wayne, Fort Wayne, IN 46809, USA.

Email: marshalj@pfw.edu

\begin{abstract}
Ash (Fraxinus spp.) is one of the most widely distributed tree genera in North America. Populations of ash in the United States and Canada have been decimated by the introduced pest Agrilus planipennis (Coleoptera: Buprestidae; emerald ash borer), having negative impacts on both forest ecosystems and economic interests. The majority of trees succumb to attack by A. planipennis, but some trees have been found to be tolerant to infestation despite years of exposure. Restriction site-associated DNA (RAD) sequencing was used to sequence ash individuals, both tolerant and susceptible to $A$. planipennis attack, in order to identify single nucleotide polymorphism (SNP) patterns related to tolerance and health declines. de novo SNPs were called using SAMtools and, after filtering criteria were implemented, a set of 17,807 SNPs were generated. Principal component analysis (PCA) of SNPs aligned individual trees into clusters related to geography; however, five tolerant trees clustered together despite geographic location. A subset of 32 outlier SNPs identified within this group, as well as a subset of 17 SNPs identified based on vigor rating, are potential candidates for the selection of host tolerance. Understanding the mechanisms of host tolerance through genome-wide association has the potential to restore populations with cultivars that are able to withstand A. planipennis infestation. This study was successful in using RAD-sequencing in order to identify SNPs that could contribute to tolerance of $A$. planipennis. This was a first step toward uncovering the genetic basis for host tolerance to A. planipennis. Future studies are needed to identify the functionality of the loci where these SNPs occur and how they may be related to tolerance of $A$. planipennis attack.
\end{abstract}

KEYWORDS

ash, emerald ash borer, RAD-seq, survival, tolerance 


\section{1 | INTRODUCTION}

Agrilus planipennis Fairmaire (Coleoptera: Buprestidae; emerald ash borer) is a metallic green beetle native to northeastern Asia that has become a pest to North American ash (Fraxinus spp. L.; McCullough et al., 2004). This pest was introduced into the Detroit/ Windsor area of Michigan, USA/Ontario, Canada, and quickly dispersed via human assistance, including movement of firewood, nursery stock, and wood packing material (Buck \& Marshall, 2008; Cappaert et al., 2005). In its native range, A. planipennis coevolved with Manchurian ash (F. mandshurica Rupr.) and is a secondary pest in this tree species, requiring a primary stressor for successful attack (Rebek et al., 2008; Whitehill et al., 2012). Kelly et al. (2020) confirmed that Asian ash species occur in distinct phylogenetic lineages with candidate genes for tolerance. Ash species in North America lack this natural resistance and succumb to attack, regardless of the presence of a primary stressor, often within one to four years after initial attack (Eyles et al., 2007; Rebek et al., 2008). While black (F. nigra Marsh.), green (F. pennsylvanica Marsh.), and white ( $F$. americana L.) ash are the most susceptible in the introduced range of A. planipennis, all North American ash are susceptible (Anulewicz et al., 2006; Cappaert et al., 2005; Poland \& McCullough, 2006). Blue ash ( $F$. quadrangulata Michx.) has the lowest susceptibility to A. planipennis attack among North American ash species (Tanis \& McCullough, 2012).

In this introduced range, the life cycle of $A$. planipennis is typically completed within one year (Herms \& McCullough, 2014). Beginning in May, adults emerge through D-shaped exit holes and mature within seven days as they feed on canopy leaves. Males identify suitable mates via visual and contact cues, and females feed on foliage for an additional five to seven days after mating before oviposition begins (Lelito et al., 2007, 2009; Poland \& McCullough, 2006). During oviposition, females deposit eggs in bark cracks and crevices, where eggs hatch within two weeks, and larvae tunnel into the bark to feed on the phloem and vascular cambium of the tree from late summer to autumn. Phloem consumption creates serpentine-shaped galleries, which severs photosynthate transport leading to eventual tree mortality. After completing four instars, larvae overwinter in pupal chambers and pupation occurs the following spring (Herms \& McCullough, 2014; Poland \& McCullough, 2006). While a one-year life cycle is most common, a two-year life cycle does occur, especially in more northern latitudes, with larvae overwintering in intermediate instars within the phloem (Wei et al., 2007).

The proliferation of A. planipennis throughout forests in North America has caused the mortality of millions of ash trees, producing devastating ecological and economic impacts (Flower et al., 2013; Kovacs et al., 2011; Poland \& McCullough, 2006). These impacts have created long-lasting changes to North American forest ecosystems and may require substantial restoration efforts (Herms \& McCullough, 2014; Marshall, 2020; Poland \& McCullough, 2006). Additional negative impacts include reduction in amount of wood products produced from ash and diminished esthetics in urban and suburban neighborhoods (Flower et al., 2013; Poland \&
McCullough, 2006). The cost of removal and replacement of ash trees in urban landscapes has been estimated at $\$ 12.5$ billion from 2010 to 2020 (Kovacs et al., 2011). Additionally, the estimated loss by timberlands in the United States is $\$ 300$ billion (Poland \& McCullough, 2006).

Given the large-scale distribution of $A$. planipennis, management options for control of the pest remain limited, which is further complicated by natural and urban landscapes. Therefore, a long-term solution to preserving ash will depend on successfully identifying resistant or tolerant populations. Resistance to wood-boring beetles is typically a function of female host selection and larval survival rate (Hanks, 1999). Therefore, resistance mechanisms can be placed into three general categories: antixenosis, antibiosis, and tolerance (Kogan \& Ortman, 1978; Painter, 1951). Antixenosis traits are aimed at decreasing preferences for feeding and/or ovipositioning, while antibiosis results from traits that negatively affect insect growth, survival, and/or fecundity. Lastly, tolerance is the ability of the host to withstand infestation while remaining relatively healthy compared to other individuals undergoing the same level of attack.

There is evidence of antixenotic traits in the interaction between A. planipennis and hosts. Adults of A. planipennis express variation in both feeding and oviposition host preferences. When given a choice, adult beetles preferentially feed on white, green, and black ash compared with Manchurian, blue, and European ash (F. excelsior L.; Pureswaran \& Poland, 2009). North American ash species receive more eggs compared with Manchurian ash, suggesting a female choice of susceptible hosts in order to increase larval performance (Gripenberg et al., 2010; Rigsby et al., 2014). Within North American ash species, inter- and intraspecific variation of volatile emissions and oviposition preferences of $A$. planipennis have been shown to play a role in resistance (Anulewicz et al., 2008; Chen et al., 2011; Koch et al., 2015). Bark of blue ash has a phenolic composition that may contribute to its resistance relative to white, green, and black ash (Whitehill et al., 2012). Bark smoothness, as a physical characteristic, may be a limiting factor in oviposition locations and subsequently limits the number of larvae that could attack a tree at a given time (Marshall et al., 2013). Additionally, variability in ash growth rates has been related to susceptibility to $A$. planipennis, with trees tolerant of attack having more rapid and constant growth compared to susceptible trees (Boyes et al., 2019).

Antibiosis interactions also exist in larval development. Mechanisms that affect larval performance mainly focus on variation in phenolic and defense protein chemistry (Cipollini et al., 2011; Villari et al., 2016; Whitehill et al., 2011, 2012). Previous studies comparing phenolic and lignin profiles of ash species found that Manchurian ash contains unique profiles that may contribute to their resistance to A. planipennis (Cipollini et al., 2011; Whitehill et al., 2011, 2012). Four potential defense-related proteins are expressed more than fivefold higher in Manchurian ash than in other species and may contribute to resistance (Whitehill et al., 2011).

Mechanisms of tolerance are more difficult to quantify and therefore have not been as well studied. Identifying the genetic variants that allow these surviving trees in North America to tolerate 
infestation would greatly aide in the conservation of ash (Villari et al., 2016). Even with severe levels of ash mortality in the introduced range, certain trees have been able to survive after years of repeated exposure (Marshall et al., 2013). This has led to the identification of trees with differing apparent tolerance levels to A. planipennis attack. Trees classified as tolerant survive in spite of signs of A. planipennis attack and damage (Marshall et al., 2013). The objectives of this study were to (1) identify ash single nucleotide polymorphisms associated with the tolerance-susceptibility gradient to A. planipennis, (2) identify phenotypic and genotypic relationships between trees relative to this tolerance-susceptibility gradient, and (3) test the hypothesis that tolerance and susceptibility are linked to identifiable genetic markers.

\section{2 | METHODS}

\section{1 | Study species and sample collection}

Trees were selected from Fraxinus spp. individuals within Fort Wayne, Indiana, USA ( $n=3)$; Huron-Clinton Metroparks, Michigan, USA (Kensington, Lower Huron, Oakwoods, and Willow; $n=39$ ); and Houghton County, Michigan, USA ( $n=5$; Figure 1). All individuals were naturally occurring and not planted. Within most of these locations, green ash was the dominant species with white ash being less common. However, in Houghton County, white ash dominated. Selection of trees was based on their occurrence along an apparent conceptual gradient from high tolerance to high susceptibility (i.e., low tolerance) to A. planipennis attack, similar to a gradient described by Hietala (2013). Apical buds were collected from trees and placed in liquid nitrogen immediately after collection. Fort Wayne and Huron-Clinton Metropark collections were made in July 2014. Houghton County collections were made in August 2016. Once returned to the laboratory, samples were stored at $-80^{\circ} \mathrm{C}$.

\section{2 | Tree assessment}

Selected trees were identified to species and assessed on vigor (overall tree health: categorical 1-5 with 1 being high vigor [crown with relatively few dead twigs; normal foliage color and density] and 5 being low vigor [more than half of crown dead]), crown dieback (percent of dead branch tips: $5 \%-100 \%$ ), and signs of A. planipennis attack (presence/absence: bark splits, exit holes, woodpecker damage, epicormic sprouts). Assessments followed those conducted in previous studies (Clark et al., 2015; Marshall et al., 2009, 2010, 2012, 2013), which were derived from Millers et al. (1991). After assessment, 47 individuals were selected for analysis and given an overall categorization of tolerant or susceptible to A. planipennis infestation. This tolerant-susceptible categorization was similar to Hietala (2013). Individuals with a vigor $\leq 3$ and dieback of $\leq 30 \%$ were considered tolerant. Individuals with a vigor of $\geq 3$ and dieback $>30 \%$ were considered susceptible. Chi-square analysis was used to test the null hypothesis that tolerance categorization was independent of species. Additionally, diameter at breast height (dbh, $1.37 \mathrm{~m}$ above soil surface) was collected as a size metric and compared between species and tolerance groups using a two-way analysis of variance (ANOVA).

\section{3 | DNA extraction and quantification}

Entire bud samples (two to three buds) were homogenized using sterile ceramic mortars and pestles, which were first cooled with liquid nitrogen. DNeasy Plant Mini Kit (QIAGEN) was used to extract total genomic DNA following the manufacturer's protocol. DNA from each sample was quantified using UV spectrophotometry (NanoDrop 1000) absorbance. All samples were subsequently diluted to a concentration of $25 \mathrm{ng} / \mu \mathrm{l}$.

\subsection{Library creation and SNP discovery}

Genomic DNA was converted into nextRAD genotyping-bysequencing libraries (SNPsaurus, LLC) as described by Russello et al. (2015). Briefly, genomic DNA was first fragmented with Nextera reagent (Illumina, Inc.), which also ligates short adapter sequences to the ends of the fragments. The Nextera reaction was scaled for fragmenting 7 ng of genomic DNA, although 14 ng of genomic DNA was used for input to compensate for the amount of degraded DNA in the samples and to increase fragment sizes. Fragmented DNA was then amplified for 25 cycles at $75^{\circ} \mathrm{C}$, with one of the primers matching the adapter and extending eight nucleotides into the genomic DNA with the selective sequence TGCAGGAG. Thus, only fragments starting with a sequence that can be hybridized by the selective sequence of the primer were efficiently amplified. The nextRAD libraries were sequenced on a HiSeq 4000 with one lane of 150 bp reads (University of Oregon).

The genotyping analysis used custom scripts (SNPsaurus, LLC) that trimmed the reads using bbduk (BBMap tools, http://sourc eforge.net/projects/bbmap/). Command was as follows:

bash bbmap/bbduk.sh in $=\$$ file out $=\$$ outfile ktrim $=r \quad k=17$ hdist=1 mink=8 ref=bbmap/resources/nextera.fa.gz minlen=100 ow $=$ t qtrim $=r$ trimq $=10$

Next, a de novo reference genome was created by collecting 10 million reads in total, evenly from the samples, and excluding reads that had counts fewer than 7 or more than 700 . The remaining loci were then aligned to each other to identify allelic loci and collapse allelic haplotypes to a single representative. All reads were mapped to the reference with an alignment identity threshold of $95 \%$ using bbmap (BBMap tools). In order to assess the proportion of sequence reads that originated from Fraxinus spp. versus other species, 1,000 high-quality reads from each sample were subject to BLASTN analysis in the NCBI database.

Genotype calling was done using SAMtools and BCFtools ( $\mathrm{Li}$ et al., 2009). Command was as follows: 

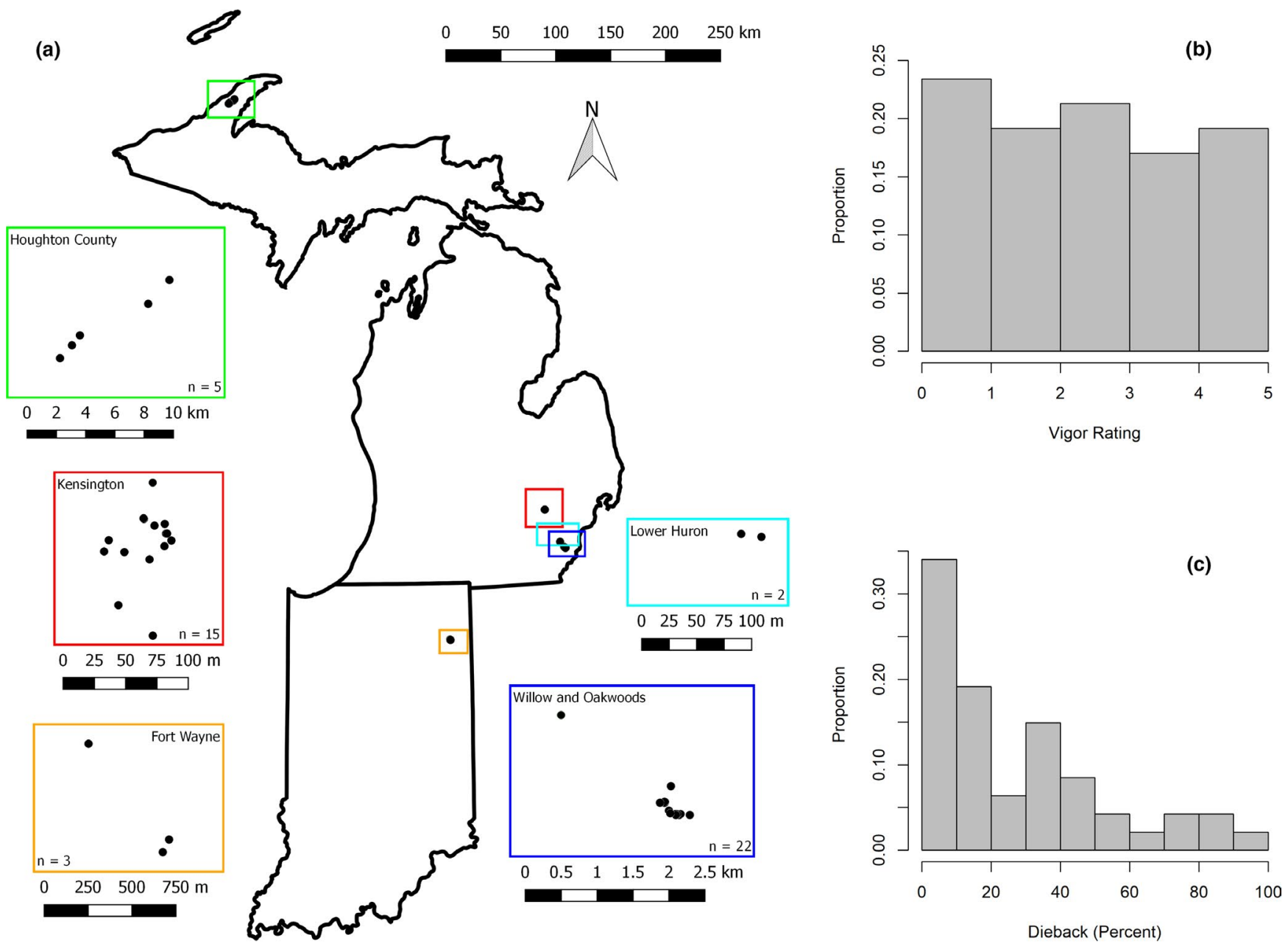

FIG URE 1 Location of sampled trees in Fort Wayne, Indiana, USA (a); Huron-Clinton Metroparks, Michigan, USA (Kensington, Lower Huron, Oakwoods, and Willow); and Houghton County, Michigan, USA. Proportion of trees per category of vigor (b) and dieback (c) determined by field assessments

TABLE 1 Filtering criteria for polymorphic loci

\begin{tabular}{|ll|}
\hline Filtering Criteria & Quantification \\
\hline Heterozygous in all samples & False \\
\hline More than 2 alleles in a sample & False \\
\hline Phred-like quality score & $>20$ \\
\hline Minor allele frequency & $>0.1$ \\
\hline Maximum missing genotype & 0.5 \\
\hline Minimum mean read depth & 14 \\
\hline
\end{tabular}

Note: Loci failing to meet the quantification threshold for any of the criteria were excluded from subsequent analysis.

samtools mpileup -gu -Q 12 -t DP, DPR -f ref.fasta -b samples.txt | bcftools call -cv - > genotypes.vcf

The VCF file was filtered to remove alleles with a population frequency of less than 0.03 . Loci were removed that were heterozygous in all samples or had more than two alleles in a sample (suggesting collapsed paralogs). The absence of artifacts was checked by counting single nucleotide polymorphisms (SNPs) at each read nucleotide position and determining that SNP number did not increase with reduced base quality at the end of the read. All polymorphic sequences retained were subject to BLASTN analysis in the NCBI database.

VCFtools (Danecek et al., 2011) was used to further filter SNPs based on the following criteria: (1) Phred quality score, (2) minor allele frequency, (3) maximum missing genotype, and (4) minimum mean read depth (Table 1). Loci that failed to meet the quantification threshold for any of the filtering criteria were removed and excluded from subsequent analyses. Samples were not filtered based on HardyWeinberg expectations because the goal of this study was to identify polymorphic loci under selection, which are expected to deviate from equilibrium. The VCF file was converted into file formats necessary for analysis using PGDSpider 2.1.1.3 (Lischer \& Excoffier, 2012).

\section{5 | SNP variation}

PLINK v 1.07 (Purcell et al., 2007) was used to assess stratification by phenotype within the filtered SNP data. Initially, phenotypes were set as coded species (green ash $=1$ [control], white ash $=2$ [case]). 
Using a permutation test of pairwise identity-by-state (IBS) distance, we tested the hypothesis that green and white ash individuals were less similar between than within putative species. Phenotypes were then coded as tolerance groups (tolerant $=1$ [control], susceptible $=2$ [case]). The pairwise permutation test of IBS was used to test the hypothesis that tolerant and susceptible individuals were less similar. Pairwise IBS distance tests were run with default 100,000 permutations. All PLINK functions included the --allow-no-sex option as both green and white ash are dioecious.

The packages $v c f R$ v1.7.0 and adegenet v2.1.1 in R v3.4.2 (Jombart, 2008; Knaus \& Grünwald, 2017; R Core Team, 2017) were used to perform an individual-based principal component analysis (PCA) to characterize structure based on SNP variation. PCA was also used to visualize relationships between individuals based on phenotypic characteristics (vigor, dieback, and signs of A. planipennis attack) using prcomp R base function. Pearson's correlation was used to test the hypothesis that the first principal component axis for SNP PCA and the first principal component axis for phenotypic PCA had a linear relationship. Similarly, linear correlations were tested between the first principal component axis for SNP PCA with vigor and dieback values.

\subsection{Detection of markers under selection}

BAYESCAN v2.1 (Foll \& Gaggiotti, 2008) was used to identify outlier loci based on populations defined by the PCA clusters, as well as populations defined by vigor rating. BAYESCAN uses a hierarchical Bayesian method to estimate population-specific $F_{S T}$ coefficients as a fixation index, described by Beaumont and Balding (2004). A more conservative neutral model available in BAYESCAN (prior odds $=1,000$ ) was used to minimize the number of false positives. Prior odds or prior probability is the likelihood of the null hypothesis being true before the test is performed. This increase in prior odds corresponds to the selection model being 1,000 times less likely than the neutral model, which was a more appropriate assumption given the number of SNPs included in this analysis (Lotterhos \& Whitlock, 2014). After 100,000 iterations, SNPs with a posterior distribution over 0.95 were considered outliers. High $F_{S T}$ values (outliers) suggest that the locus has undergone directional selection (in contrast to balancing selection). Fisher's exact test was used in PLINK v1.07 (Purcell et al., 2007) to test the association of above identified outliers with the tolerance groups.

\section{3 | RESULTS}

\section{1 | Phenotypic classification}

The 47 ash trees selected for this study were sampled from six different geographic locations (Figure 1a). Based on morphological characteristics, nine trees were identified as white ash and the remaining trees were identified as green ash. The trees were classified into two major groups: tolerant or susceptible to A. planipennis attack based on both vigor and dieback ratings. This categorization resulted in $\mathbf{2 8}$ trees being classified as tolerant and 19 as susceptible. Vigor rating categories were evenly represented within each category (Figure 1b). However, dieback was skewed right, with fewer trees having greater values of dieback (Figure 1c). Overall, signs of $A$. planipennis infestation (i.e., bark splits, exit holes, woodpecker damage, epicormic sprouts) were present in 37 individuals, 31 of which had more than one sign present. Of the ten trees lacking signs, eight were categorized as tolerant and two were categorized as susceptible. There was a significant difference in dbh between species $(F=6.19, d f=1,43, p$-value $=.017)$ with white being larger than green. However, there was no difference in dbh among tolerance groups $(F=0.40, d f=1,43, p$-value $=.531)$ and the interaction between species and tolerance groups was not significant $(F=0.58$, $d f=1,43, p$-value $=.449$ ).

\section{2 | RAD-sequencing}

Restriction site-associated DNA sequencing was used to sequence the genomes of 47 ash trees in order to identify SNPs that are correlated with tolerant or susceptible phenotypes. BLASTN analysis of the 1,000 random sequences from each individual revealed that $60.7 \%$ of the genomic sequences were potentially ash-specific (no hits discovered). A high percentage of mitochondrial reads (18.0\%) was found, potentially diverting reads from nuclear loci. Of sequences

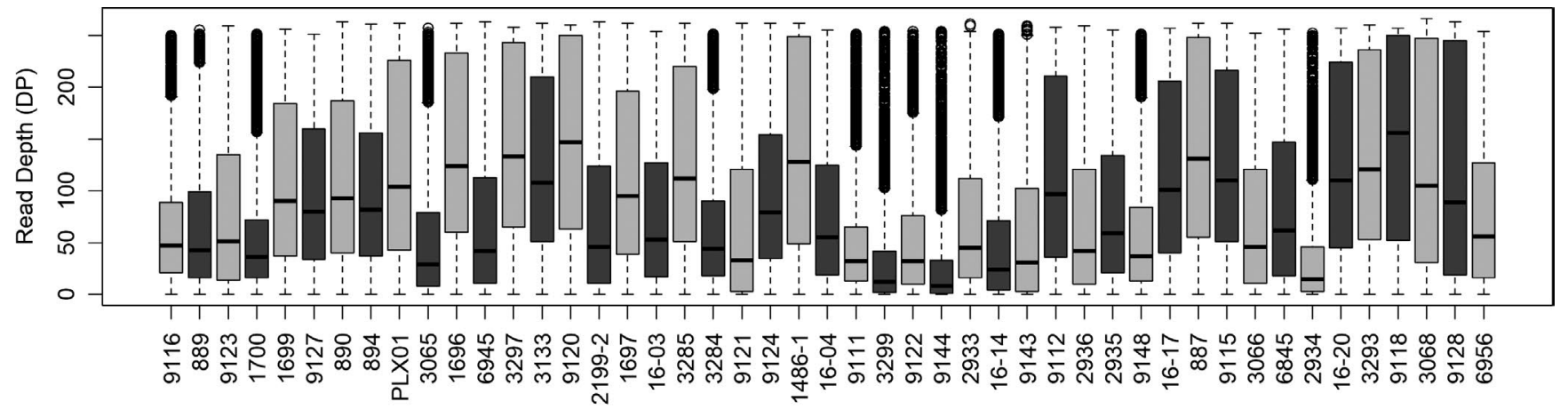

FIGURE 2 Boxplot of read depth for all sampled ash individuals 
mapped, $17.7 \%$ aligned to Populus tremula, 0.5\% Sesamum spp., and $0.5 \%$ Olea europaea. Due to the high mitochondrial hit counts, a reference of just polymorphic loci was created and BLASTN analysis was performed on those sequences. Out of this entire subset, only eight loci mapped to mitochondrial sequences.

After SNP calling and initial filtering, a set of 23,243 SNPs were produced. Application of the more stringent filtering criteria (Table 1) generated a final set of 17,807 SNPs. Individuals were assessed for read depth. Mean read depth over all individuals was 91.5, and means ranged from 30 to 150 (Figure 2).

\section{3 | SNP variation}

Pairwise IBS distance test to compare SNP similarity between species (green and white) failed to reject the hypothesis that pairs between the two species were less similar than pairs within species $(p$-value $=.240)$. This lack of variability between pairs extended into the other pairwise tests, with green pairs not more similar than white pairs ( $p$-value $=.756$ ). Additionally, the pairs between tolerance groups (tolerant and susceptible) were not less similar than pairs within groups ( $p$-value $=.620$ ). However, susceptible pairs were more similar than tolerant pairs ( $p$-value $=.027$ ).

Four major clusters (labeled as right, lower, upper, and middle) were identified in the PCA based on 17,807 SNPs (Figure 3a). Diverging substantially from all other groups, the cluster on the right contained trees from different geographic locations, including Houghton and three Metroparks (Kensington, Oakwoods, and Willow). Furthermore, all the individuals in this cluster were classified as tolerant to A. planipennis attack (Figure 3a). IBS clustering resulted in a multidimensional scaling ordination plot that did not differ from the visual clusters present in the PCA ordination plot.

\subsection{Outlier SNPs}

Outlier detection based on the four PCA clusters identified 32 outlier SNPs within 28 different loci (Table 2). Of these outliers detected with the PCA clusters, nine were significantly associated with the tolerant group. Outlier detection based on vigor rating identified 17 outlier SNPs with 13 different loci (Table 3). Of these outliers detected with vigor rating, five were significantly associated with the tolerant group and five were significantly associated with the susceptible group (Table 3 ). All outlier $F_{S T}$ values were skewed to the right and outliers were relatively high, suggesting directional selection (Figure 4). There were no outliers detected by analysis when trees were grouped based on tolerance and susceptibility. Out of all 41 outlier loci, only one matched to a known sequence from the NCBI nucleotide database (locus 30133_137; top blast hit: XM_011468015.1). This locus mapped to a PTI1-like tyrosineprotein kinase receptor.

Of the outliers detected between the PCA clusters, ten had a clear pattern of the polymorphic nucleotide being predominantly present in the five right cluster individuals (Figure 5). These patterns were slightly offset by similar genetic trends between the middle and right clusters; however, the right cluster clearly had the highest occurrence of these polymorphisms. Interestingly, one set of outlier SNPs, all occurring at the locus 16669_22, was present in all trees except the five in the right group and two trees from the middle group (Figure 6). These trees retained the reference nucleotide in
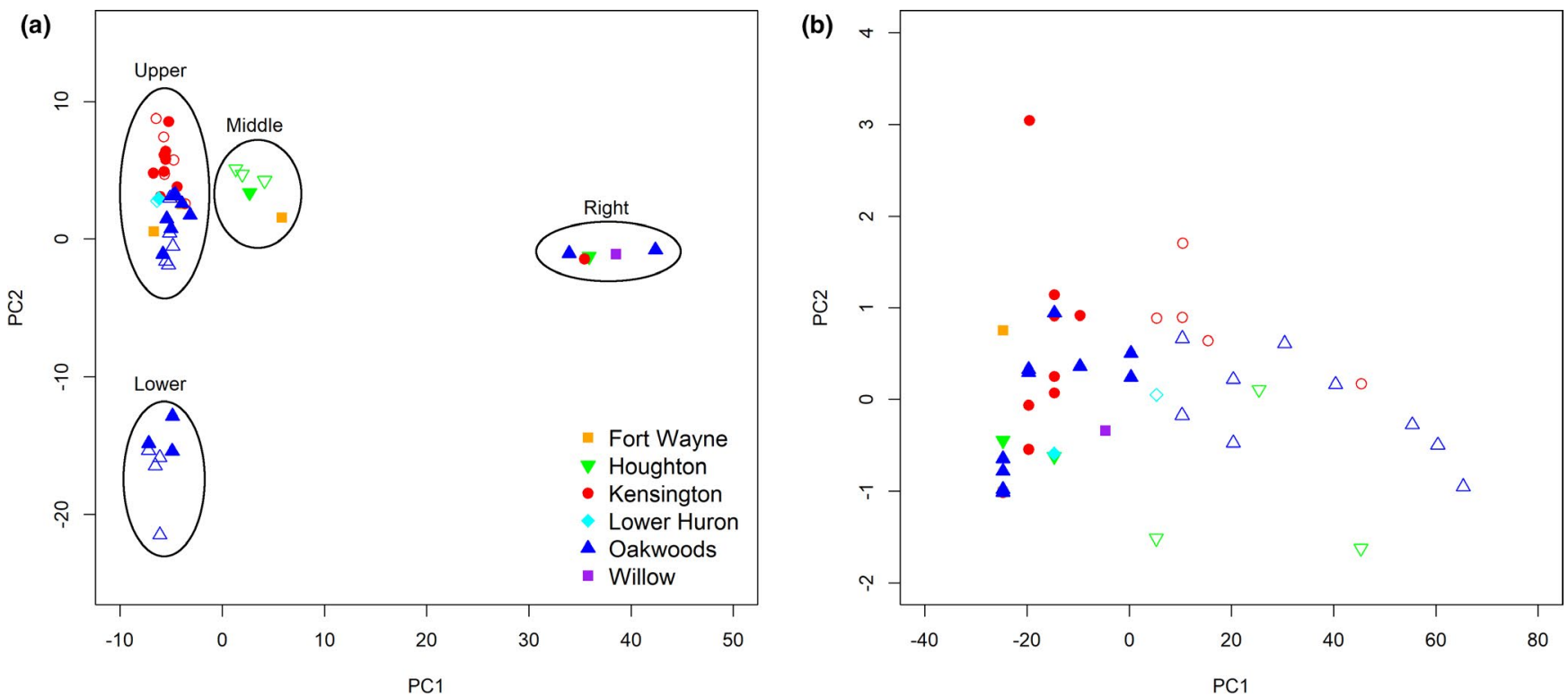

FIGURE 3 Principal component analysis (PCA) of filtered single nucleotide polymorphisms (SNPs) (a) and phenotypic data (b) from ash individuals in Fort Wayne, Indiana, USA; Huron-Clinton Metroparks, Michigan, USA (Kensington, Lower Huron, Oakwoods, and Willow); and Houghton County, Michigan, USA. Closed symbols represent tolerant trees and open symbols represent susceptible trees. Ellipses are arbitrary, represent visual clustering, and are presented for labeling of cluster locations in PCA 
TABLE 2 Summary of outlier loci detected between four clusters within PCA

\begin{tabular}{|c|c|c|c|c|c|c|c|}
\hline Locus & $\begin{array}{l}\text { SNP } \\
\text { Location }\end{array}$ & SNP & $F_{S T}$ & $q$ Value & Tolerant & Susceptible & $p$-Value \\
\hline 2690_69 & 86 & $\mathrm{G} / \mathrm{A}$ & 0.472 & 0.045 & 0.21 & 0.00 & .003 \\
\hline 6512_10 & 66 & $A / G$ & 0.472 & 0.042 & 0.22 & 0.03 & .012 \\
\hline 8409_8 & 142 & $A / G$ & 0.533 & 0.002 & 0.25 & 0.38 & .314 \\
\hline 13284_148 & 49 & $\mathrm{G} / \mathrm{A}$ & 0.504 & 0.031 & 0.15 & 0.07 & .470 \\
\hline 13980_8 & 103 & $\mathrm{~T} / \mathrm{C}$ & 0.505 & 0.006 & 0.17 & 0.03 & .043 \\
\hline 16669_22 & 10 & $\mathrm{~T} / \mathrm{C}$ & 0.532 & 0.004 & 0.26 & 0.12 & .145 \\
\hline 16669_22 & 13 & $A / G$ & 0.532 & 0.003 & 0.26 & 0.12 & .145 \\
\hline 18944_8 & 106 & $\mathrm{~T} / \mathrm{C}$ & 0.437 & 0.05 & 0.23 & 0.22 & 1.000 \\
\hline 22135_11 & 3 & $\mathrm{~T} / \mathrm{A}$ & 0.476 & 0.008 & 0.29 & 0.11 & .042 \\
\hline 24473_11 & 46 & T/A & 0.505 & 0.002 & 0.33 & 0.08 & .005 \\
\hline 30133_137 & 109 & $\mathrm{G} / \mathrm{A}$ & 0.486 & 0.014 & 0.22 & 0.06 & .039 \\
\hline 31830_23 & 118 & $\mathrm{C} / \mathrm{T}$ & 0.479 & 0.026 & 0.27 & 0.00 & .001 \\
\hline 34843_20 & 109 & $\mathrm{G} / \mathrm{A}$ & 0.465 & 0.007 & 0.45 & 0.50 & .806 \\
\hline 37961_17 & 133 & $\mathrm{C} / \mathrm{T}$ & 0.531 & 0.001 & 0.48 & 0.34 & .263 \\
\hline 38762_25 & 57 & $\mathrm{G} / \mathrm{A}$ & 0.474 & 0.035 & 0.54 & 0.33 & .102 \\
\hline 57187_42 & 84 & $\mathrm{G} / \mathrm{A}$ & 0.536 & 0.003 & 0.40 & 0.19 & .054 \\
\hline 59640_10 & 101 & $\mathrm{G} / \mathrm{A}$ & 0.506 & 0.005 & 0.28 & 0.13 & .151 \\
\hline 61718_43 & 96 & $\mathrm{~T} / \mathrm{C}$ & 0.485 & 0.022 & 0.17 & 0.06 & .284 \\
\hline 67007_71 & 5 & $\mathrm{C} / \mathrm{A}$ & 0.461 & 0.017 & 0.40 & 0.34 & .636 \\
\hline 88261_24 & 115 & $\mathrm{~T} / \mathrm{C}$ & 0.51 & 0.004 & 0.26 & 0.13 & .253 \\
\hline
\end{tabular}

Note: Includes single nucleotide polymorphism (SNP) location within loci, reference/polymorphic nucleotide pairs, and $F_{S T}$ and $q$ Values. False discovery rate of $<0.05$ was used. Frequency in tolerant and susceptible groups from association Fisher's exact test, with $p$-value.

this case, not the polymorphic nucleotide. For the outlier SNPs present at this locus, each individual either had all four polymorphic nucleotides or retained all four reference nucleotides.

Of the outliers detected between groups based on vigor rating, four had a clear pattern of the polymorphic nucleotide being present exclusively in trees with high vigor (Figure 7). Three of these SNPs were present at one locus (4467_128). One outlier SNP at locus 10225_13 displayed a pattern of the reference nucleotide occurring more frequently in trees with high vigor (i.e., low vigor rating values), whereas trees with low vigor (i.e., high vigor rating values) all had the polymorphic nucleotide (Figure 8).

\section{5 | Phenotypic analysis}

PCA based on all phenotypic data resulted in no distinct clustering between geographic locations (Figure $3 b$ ). Tolerant and susceptible trees did separate in this PCA; however, this is due to the categorization being defined by the same phenotypic data used to calculate the PCA. The PC1 axes of both the SNP and phenotype PCA analyses were not correlated $(r=-0.16, p$-value $=.270)$. There was no correlation between the SNP PC1 axis and vigor $(r=-0.22, p$ value $=.132$ ). Likewise, there was no correlation between the SNP PC1 axis and dieback $(r=-0.16, p$-value $=.271)$. 
TABLE 3 Summary of outlier loci detected between populations based on vigor rating

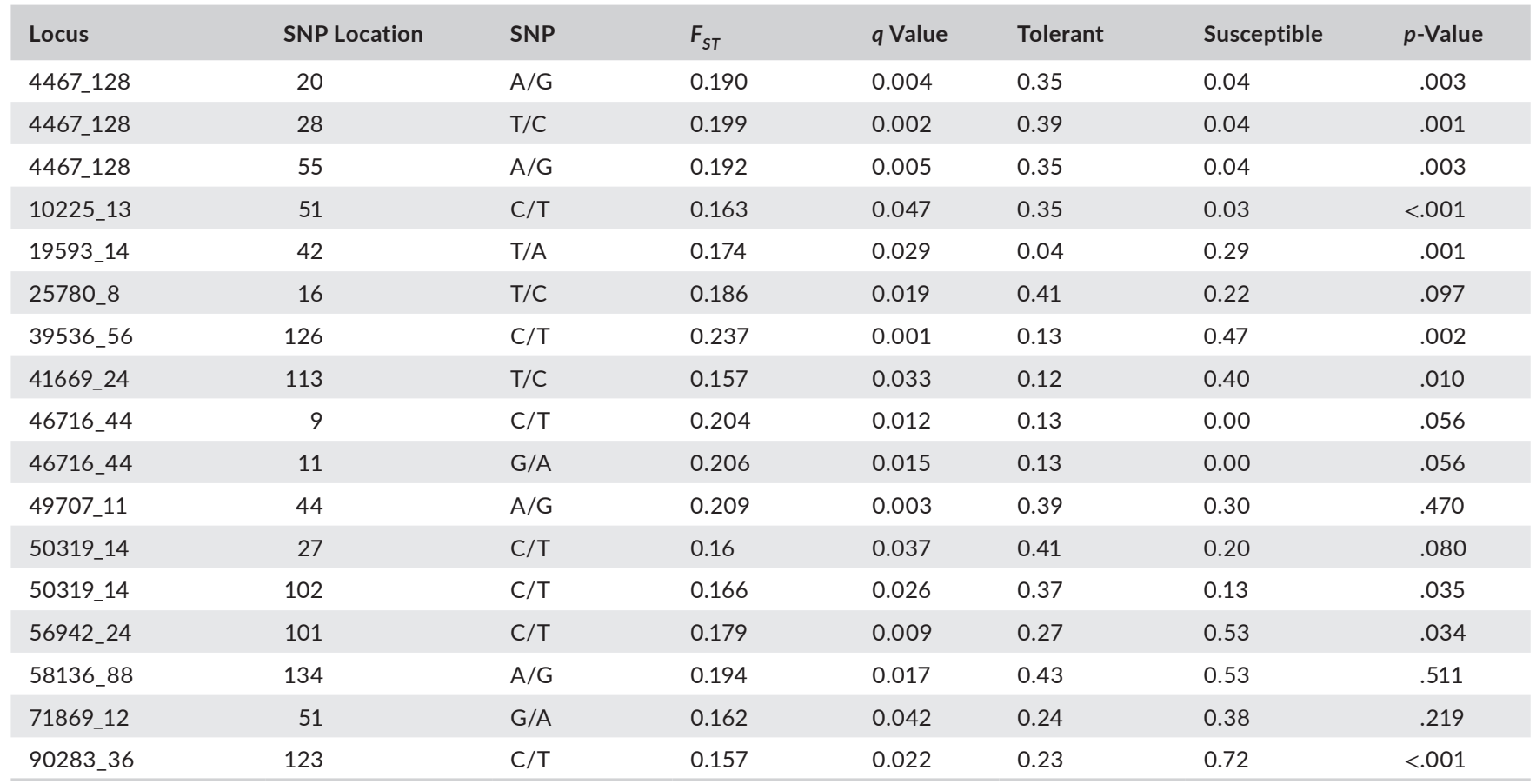

Note: Includes single nucleotide polymorphism (SNP) location within loci, reference/polymorphic nucleotide pairs, and $F_{S T}$ and $q$ Values. False discovery rate of $<0.05$ was used. Frequency in tolerant and susceptible groups from association Fisher's exact test, with $p$-value.

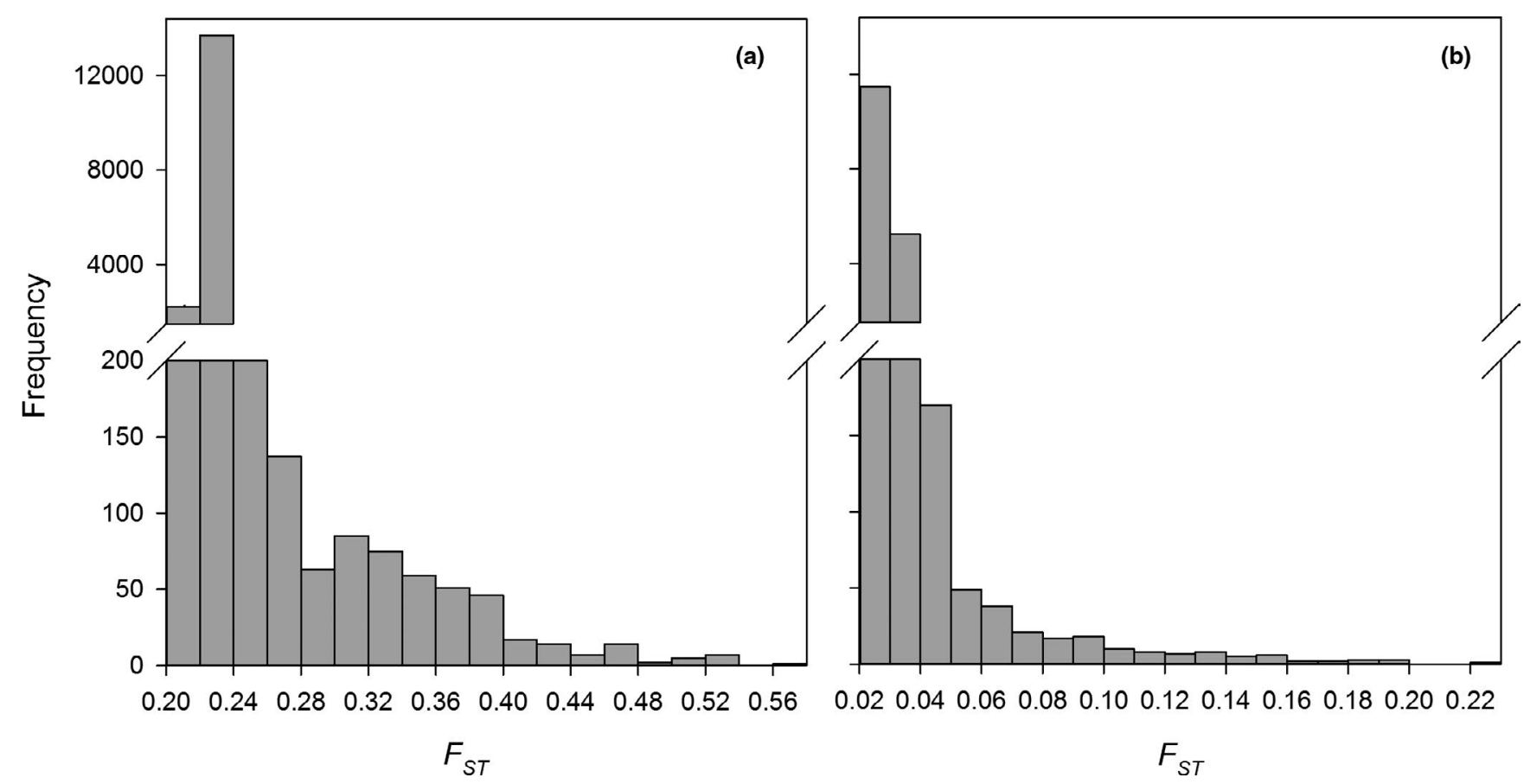

FIGURE 4 Frequency of $F_{\mathrm{ST}}$ values of all SNPs computed between populations based on the PCA clusters (a) and populations based on vigor rating (b)

\section{DISCUSSION}

This study identified polymorphic loci in Fraxinus spp. using RADsequencing genotyping-by-sequencing. The filter settings were selected to ensured high-quality nucleotide data with sufficient coverage across individuals (Bao et al., 2014; Nielsen et al., 2011). The resulting SNPs were used to highlight insights into a potential genetic basis for host tolerance to A. planipennis.

While the two species were different in regard to mean dbh, there no differences between the two tolerance groups. Size is 
FIGURE 5 Proportion of trees in each PCA cluster that had the polymorphic nucleotide (white bars) or the reference nucleotide (black bars) for the ten outlier loci identified as having a clear presence in the right group. Within each locus, there was only one outlier SNP identified. Sample sizes for each cluster were as follows: right $(n=5)$, lower $(n=7)$, upper $(n=30)$, middle $(n=5)$. Samples with missing data for that loci were not included
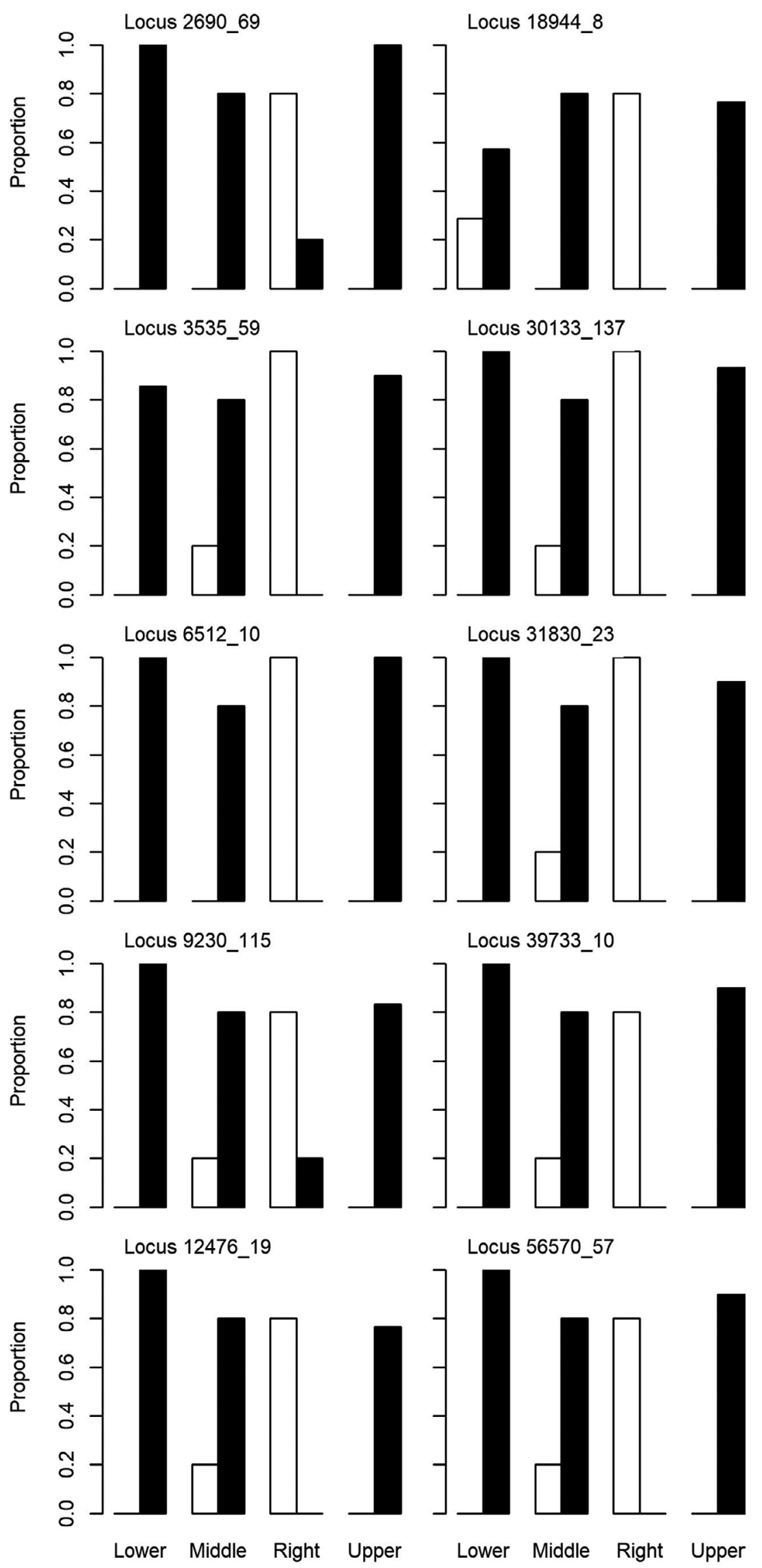

Cluster

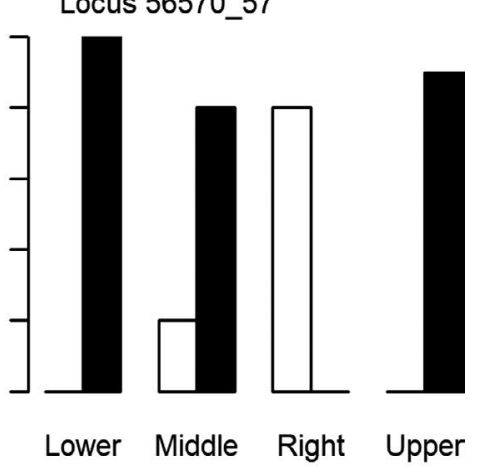

Cluster 
an important factor in A. planipennis infestation, with larger trees ( $\geq 25 \mathrm{~cm} \mathrm{dbh}$ ) being the most likely attacked (Marshall et al., 2009). However, size importance in subsequent mortality is not as strong, as other physiological factors (e.g., increased bark roughness, decreased growth rates) may be more important in mortality (Boyes et al., 2019; Marshall et al., 2013).

\section{1 | Patterns of genetic variation}

While the distance pairwise tests did not identify tolerance group pairs as more similar than across the two groups, there was less variation in the susceptible group pairs compared to the tolerant group pairs. PCA plots provided visual representation of genetic divergence among individuals. The PCA adds to the quantitative pairwise analysis in that visual clusters contained both tolerant and susceptible individuals, except for the cluster labeled "Right." There was no clear relationship between the PCA clusters and geographic distribution, as three of the four clusters contained trees from multiple locations.

Field identified green and white ash did not separate as expected either-qualitatively in the PCA or quantitatively in pairwise distance analysis. This is not the first occurrence of genetic overlap of these two species. White ash is a polyploid species $(2 n=46$, 92, and 138) but most often diploid similar to green ash, and hybridization appears to confound genetic results (Schlesinger, 1990; Wallander, 2008; Whittemore et al., 2018). In some cases, white ash individuals group with green ash in phylogenetic analyses, a result that was attributed to the white ash samples likely being a polyploid hybrid with green ash (Wallander, 2008). Additionally, there is low genetic differentiation between white, velvet ( $F$. velutina Torr.), and

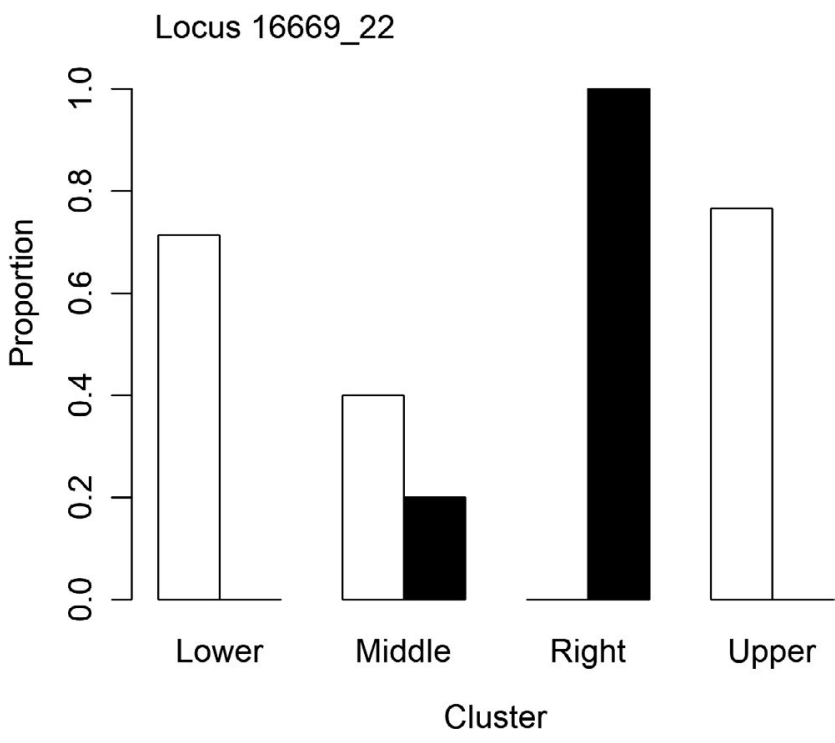

FIGURE 6 Proportion of trees in each PCA cluster that had the polymorphic nucleotide (white bars) or the reference nucleotide (black bars) for the locus 16669_22. Four SNPs were identified as outliers within this one locus (Table 3). Sample sizes for each cluster were as follows: right $(n=5)$, lower $(n=7)$, upper $(n=30)$, middle $(n=5)$. Samples with missing data were not included green ash (Hinsinger et al., 2013). Rapid radiation or recent exchange of genetic material could have led to these relationships (Hinsinger et al., 2013). This lack of interspecific variation is especially present in chloroplasts (Jeandroz et al., 1997). The co-occurrence of white and green ash in all sampling locations presents the possibility of hybridization between the two species; therefore, data were analyzed as Fraxinus spp. due to expected low genetic differentiation. Additionally, the pairwise IBS distance analysis in PLINK did not find green and white individuals were less similar, further suggesting that our SNP data were unable to separate the two species.

Across the PCA, there was little separation based on tolerance and susceptibility categories applied by field assessment data. The exception to this pattern was the right cluster, which contained five tolerant individuals from various geographic locations in Michigan. Four of those individuals were in close proximity to the de facto introduction epicenter for A. planipennis (Siegert et al., 2014), indicating they have been exposed to A. planipennis for nearly 20 years and are still able to tolerate infestation (Marshall et al., 2013). These five trees were located in Houghton County and three Metroparks (Kensington, Oakwoods, and Willow). For this reason, outlier SNPs were identified between the four clusters on the PCA to determine which SNPs were likely causing the variation in this group.

\section{2 | SNP candidates for tolerance selection}

All outlier SNPs detected between the PCA clusters had high $F_{S T}$ values and appeared to be responsible for the divergence of the right cluster. However, subsequent PCA on SNP variation with these outliers removed (results not shown) revealed that the five individuals in the right cluster still displayed the same pattern of divergence, indicating that these 28 loci are not the only source of variation within this group.

Throughout the outliers identified, there were similar genetic trends between trees in the middle and right clusters. The similarities between these two clusters are evident when looking at just the ten polymorphic loci that showed a pattern of almost exclusive presence in the right group. For seven of the ten loci, one to two trees from the middle cluster also had the polymorphic nucleotide. Two of these trees from the middle cluster were classified as susceptible; however, all of the trees from the middle group that had genotypic similarities with the five right group trees had no signs of A. planipennis attack (i.e., lacking bark spits, exit holes, woodpecker activity, and sprouting), despite being located in areas where A. planipennis is present.

The outlier locus 30133_137 mapped to a PTI1-like tyrosineprotein kinase 2 . This protein is known to be involved in growth and development, as well as defense responses (Anthony et al., 2006; Floriduz et al., 2002). PTI1 serine/threonine protein kinases were described to be key components of speck disease resistance in tomatoes by amplifying signaling pathways (Sessa et al., 2000). It is possible that this gene may also play a role in defense against $A$. planipennis by amplifying pathways necessary to tolerate infestation. 
FIGURE 7 Proportion of trees in each vigor rating that had the polymorphic nucleotide (white bars) or the reference nucleotide (black bars) for the four outlier loci identified as having a clear pattern of sizes for each group were as follows: 1 ( $n=11), 2(n=9), 3(n=10), 4(n=8), 5$ $(n=9)$. Samples with missing data were not included presence in the high vigor groups. Sample

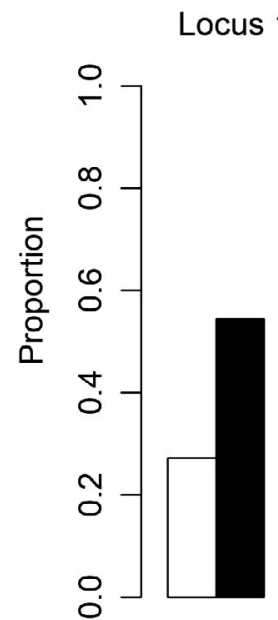

1

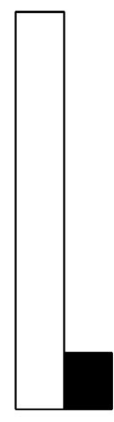

2

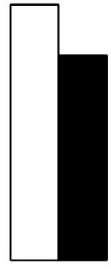

3

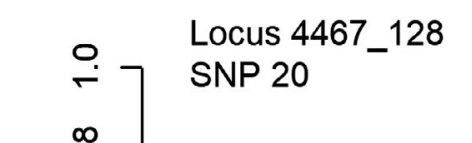

은

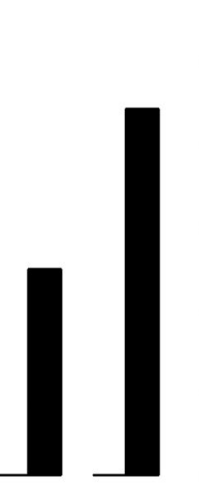

\section{Locus 4467_128 \\ SNP 55}

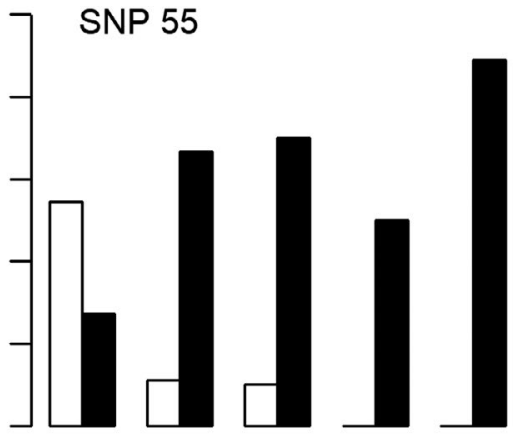

Locus 90283_36

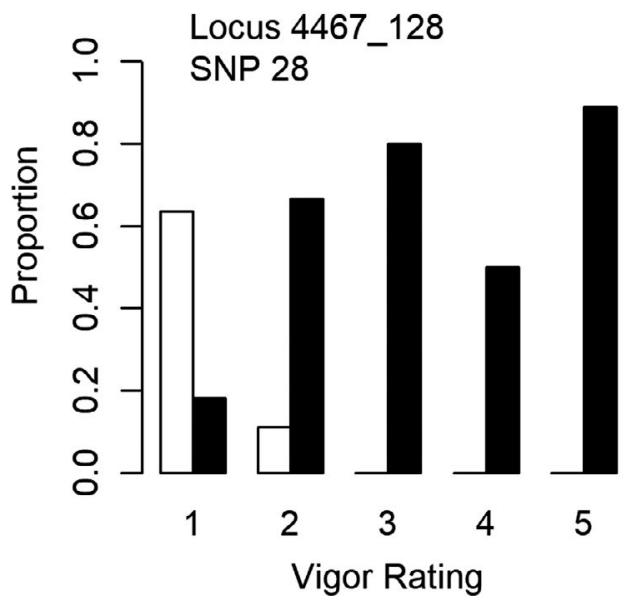

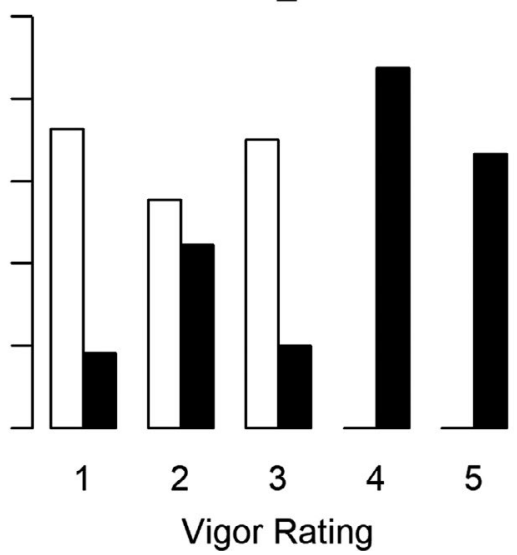

Four outlier SNPs at this locus had distinctive patterns in individuals with either all present as the polymorphism or all in their reference form. For the five individuals in the right cluster, the reference nucleotides were retained for all four SNPs at this locus. Unfortunately, BLAST analysis did not map this locus to any known genes. Additionally, two trees that clustered in the middle PCA group also had the reference nucleotides at this locus. These two trees were classified as susceptible, but interestingly, they were the only two susceptible trees with no signs of $A$. planipennis attack. This exemplifies the coarseness of categorizing tolerance based on phenotypic characteristics of vigor and dieback. These two trees with poor vigor and high dieback may have simply displayed other manifestations of decline not associated with A. planipennis (e.g., Houghton County trees were along a highway and subject to salt spray).

Outliers detected between trees based on vigor rating resulted in an additional 13 loci being identified as potential candidates for host tolerance. None of these mapped to any known functional genes; however, the five outliers that did show a pattern of either the polymorphic or the reference nucleotide being present exclusively in high vigor trees are of particular interest. With five outliers associated with each of the tolerant and susceptible groups, there may be important SNPs to identify potential host tolerance and susceptibility. One constraint to mapping sequences is the amount of recombination due to divergence and availability of a representative reference genome (Sousa \& Hey, 2013). Unmapped reads can also be attributed to conserved sequences across individuals (Gouin et al., 2015). Future analyses on characterizing the functionality of be important in identifying host tolerance and susceptibility. Locus
16669_22 is another potentially important gene for host tolerance.

From the outliers identified based on the visual PCA cluster, several were significantly associated with the tolerant group. These may
FIGURE 8 Proportion of trees in each vigor rating that had the (black bars) for the locus 10225 13. Sample sizes for each cluster were as follows: $1(n=11), 2(n=9), 3(n=10), 4(n=8), 5(n=9)$. Samples with missing data were not included 
the outlier loci detected in this study, specifically those with associations with tolerance and susceptibility, could expose the importance of these genes and the role they may play in tolerance.

A clear link between genotypic and phenotypic data was not identified. Most likely, this was due to the coarseness of phenotype classification, which then failed to correlate with complex genetic diversity. Phenotypes were defined by tree assessments, which included categorical tree health observations and presence or absence of signs of $A$. planipennis attack. A future study may have more success if these signs are quantified at finer scales (e.g., number of exit holes per square meter, area of phloem regrowth, and location of bark splits) as opposed to whole tree values. Boyes et al. (2019) demonstrated phloem regrowth in artificially damaged trees occurred at higher rates in tolerant individuals and those without exit holes. Detailed phenotypic data would allow for more robust analyses linking genotype and phenotype, such as a mixed-linear model. Finally, the power of genome-wide associations is affected by the genetic complexity and heritability of a trait (Burghardt et al., 2017). As tolerance is expected to be a complex genetic trait, this increases the chance of false positive associations. To remedy this issue in future studies, as many genotypes as possible should be used along with high-quality nucleotide data.

\section{5 | CONCLUSIONS}

Agrilus planipennis has devastated populations of Fraxinus spp. in North America; however, the survival of some individuals despite years of exposure to A. planipennis is evidence of host tolerance. Understanding the mechanisms of host tolerance through genomewide association has the potential to restore populations with cultivars that are able to persist in the presence of $A$. planipennis. Despite the caveats presented above, this study was successful in using RAD-sequencing in order to identify SNPs that are potential candidates for tolerance to A. planipennis. This was a first step toward uncovering a genetic basis for host tolerance to $A$. planipennis. Future studies are needed to identify the functionality of the outlier loci detected in this study.

\section{ACKNOWLEDGMENTS}

The authors would like to thank Nick White for field assistance and SNPsaurus, LLC, for contributing to a portion of the methods. Financial support for this work was partially provided by Purdue University Fort Wayne Institute for Research, Scholarship, and Creative Endeavors Collaborative Grant Program.

\section{CONFLICT OF INTEREST}

The authors declare no conflict of interest.

\section{AUTHOR CONTRIBUTION}

Cecelia E Hale: Conceptualization (supporting); Data curation (supporting); Formal analysis (lead); Writing-original draft (lead); Writingreview \& editing (equal). Mark Jordan: Formal analysis (supporting);
Methodology (supporting); Writing-review \& editing (supporting). Gloria Iriarte: Formal analysis (supporting); Writing-review \& editing (supporting). Kirk D Broders: Formal analysis (supporting); Writingreview \& editing (supporting). Andrew J Storer: Conceptualization (equal); Writing-review \& editing (supporting). Vamsi J Nalam: Conceptualization (lead); Data curation (lead); Formal analysis (supporting); Methodology (supporting); Writing-original draft (supporting); Writing-review \& editing (supporting). Jordan M Marshall: Conceptualization (lead); Formal analysis (supporting); Methodology (supporting); Writing-original draft (supporting); Writing-review \& editing (lead).

\section{DATA AVAILABILITY STATEMENT}

Data are available through NCBI https://www.ncbi.nlm.nih.gov/ bioproject/PRJNA561365/ and Dryad https://doi.org/10.5061/ dryad.7pvmcvdtd

\section{ORCID}

Jordan M. Marshall (iD https://orcid.org/0000-0001-6024-3758

\section{REFERENCES}

Anthony, R. G., Khan, S., Costa, J., Pais, M. S., \& Bögre, L. (2006). The Arabidopsis protein kinase PTI1-2 is activated by convergent phosphatidic acid and oxidative stress signaling pathways downstream of PDK1 and OXI1. Journal of Biological Chemistry, 281, 3753637546. https://doi.org/10.1074/jbc.M607341200

Anulewicz, A. C., McCullough, D. G., Cappaert, D. L., \& Poland, T. M. (2008) Host range of the emerald ash borer (Agrilus planipennis Fairmaire) (Coleoptera: Buprestidae) in North America: results of multiple-choice field experiments. Environmental Entomology, 37, 230-241.

Anulewicz, A. C., McCullough, D. G., \& Miller, D. L. (2006). Oviposition and development of Emerald Ash Borer (Agrilus planipennis) (Coleoptera: Buprestidae) on hosts and potential hosts in no-choice bioassays. Great Lakes Entomologist, 39, 99-112.

Bao, R., Huang, L., Andrade, J., Tan, W., Kibbe, W. A., Jiang, H., \& Feng, G. (2014). Review of current methods, applications, and data management for the bioinformatics analysis of whole exome sequencing. Cancer Informatics, 13(Suppl 2), 67-82. https://doi.org/10.4137/ CIN.S13779

Beaumont, M. A., \& Balding, E. J. (2004). Identifying adaptive genetic divergence among populations from genome scans. Molecular Ecology, 13, 969-980. https://doi.org/10.1111/j.1365-294X.2004.02125.x

Boyes, K. N., Hietala-Henschell, K. G., Barton, A. P., Storer, A. J., \& Marshall, J. M. (2019). Linking tree growth rate, damage repair, and susceptibility to a genus-specific pest infestation. Journal of Forestry Research, 30, 1935-1941. https://doi.org/10.1007/s1167 6-019-00896-y

Buck, J. H., \& Marshall, J. M. (2008). Hitchhiking as a secondary dispersal pathway for adult emerald ash borer, Agrilus planipennis. Great Lakes Entomologist, 41, 155-157.

Burghardt, L. T., Young, N. D., \& Tiffin, P. (2017). A guide to genome-wide association mapping in plants. Current Protocols in Plant Biology, 2, 22-38.

Cappaert, D., McCullough, D. G., Poland, T. M., \& Siegert, N. W. (2005). Emerald ash borer in Northern America: A research and regulatory challenge. American Entomologist, 51, 152-165.

Carpi, A., Di Maira, G., Vedovato, M., Rossi, V., Naccari, T., Floriduz, M., Terzi, M., \& Filippini, F. (2002). Comparative proteome bioinformatics: Identification of a whole complement 
of putative protein tyrosine kinases in the model flowering plant Arabidopsis thaliana. Proteomics, 2, 1494-1503. https:// doi.org/10.1002/1615-9861(200211)2:11<1494:AID-PROT1 494>3.0.CO;2-\#

Chen, Y., Whitehill, J. G., Bonello, P., \& Poland, T. M. (2011). Differential response in foliar chemistry of three ash species to emerald ash borer adult feeding. Journal of Chemical Ecology, 37, 29-39. https:// doi.org/10.1007/s10886-010-9892-1

Cipollini, D., Wang, Q., Whitehill, J. G., Powell, J. R., Bonello, P., \& Herms, D. A. (2011). Distinguishing defensive characteristics in the phloem of ash species resistant and susceptible to emerald ash borer. Journal of Chemical Ecology, 37, 450-459. https://doi.org/10.1007/ s10886-011-9954-z

Clark, R. E., Boyes, K. N., Morgan, L. E., Storer, A. J., \& Marshall, J. M. (2015). Development and assessment of ash mortality models in relation to emerald ash borer infestation. Arboriculture \& Urban Forestry, 41, 270-278. https://doi.org/10.48044/jauf.2015.025

Danecek, P., Auton, A., Abecasis, G., Albers, C. A., Banks, E., DePristo, M. A., Handsaker, R. E., Lunter, G., Marth, G. T., Sherry, S. T., McVean, G., \& Durbin, R. (2011). The variant call format and VCFtools. Bioinformatics, 27, 2156-2158. https://doi.org/10.1093/bioinforma tics/btr330

Eyles, A., Jones, W., Reidl, K., Cipollini, D., Schwartz, S., Chan, K., Herms, D. A., \& Bonello, P. (2007). Comparative phloem chemistry of Manchurian ash (Fraxinus mandshurica) and two North American ash species (Fraxinus americana and Fraxinus pennsylvanica). Journal of Chemical Ecology, 33, 1430-1448.

Flower, C. E., Knight, K. S., \& Gonzalez-Meler, M. A. (2013). Impacts of the emerald ash borer (Agrilus planipennis Fairmaire) induced ash (Fraxinus spp.) mortality on forest carbon cycling and successional dynamics in the eastern United States. Biological Invasions, 15, 931944. https://doi.org/10.1007/s10530-012-0341-7

Foll, M., \& Gaggiotti, O. (2008). A genome-scan method to identify selected loci appropriate for both dominant and codominant markers: A Bayesian perspective. Genetics, 180, 977-993. https://doi. org/10.1534/genetics.108.092221

Gouin, A., Legeai, F., Nouhaud, P., Whibley, A., Simon, J. C., \& Lemaitre, C. (2015). Whole-genome re-sequencing of non-model organisms: Lessons from unmapped reads. Heredity, 114, 494-501. https://doi. org/10.1038/hdy.2014.85

Gripenberg, S., Mayhew, P. J., Parnell, M., \& Roslin, T. (2010). A metaanalysis of preference-performance relationships in phytophagous insects. Ecology Letters, 13, 383-393. https://doi. org/10.1111/j.1461-0248.2009.01433.x

Hanks, L. M. (1999). Influence of the larval host plant on reproductive strategies of cerambycid beetles. Annual Review of Entomology, 44, 483-505. https://doi.org/10.1146/annurev.ento.44.1.483

Herms, D. A., \& McCullough, D. G. (2014). Emerald ash borer invasion of North America: History, biology, ecology, impacts and management. Annual Review of Entomology, 59, 13-30. https://doi. org/10.1146/annurev-ento-011613-162051

Hietala, K. G. (2013). Evaluation and monitoring of ash (Fraxinus spp.) tolerant to long-term emerald ash borer (Agrilus planipennis [Coleoptera: Buprestidae]) exposure. MS thesis. Michigan Technological University.

Hinsinger, D. D., Basak, J., Gaudeul, M., Cruaud, C., Bertolino, P., Frascaria-Lacoste, N., \& Bousquet, J. (2013). The phylogeny and biogeographic history of ashes (Fraxinus, Oleaceae) highlight the roles of migration and vicariance in the diversification of temperate trees. PLoS One, 8, e80431. https://doi.org/10.1371/journ al.pone.0080431

Jeandroz, S., Roy, A., \& Bousquet, J. (1997). Phylogeny and phylogeography of the circumpolar genus Fraxinus (OLEACEAE) based on internal transcribed spacer sequences of nuclear ribosomal DNA.
Molecular Phylogenetics and Evolution, 7, 241-251. https://doi. org/10.1006/mpev.1996.0393

Jombart, T. (2008). Adegenet: An R package for the multivariate analysis of genetic markers. Bioinformatics, 24, 1403-1405.

Kelly, L. J., Plumb, W. J., Carey, D. W., Mason, M. E., Cooper, E. D., Crowther, W., Whittemore, A. T., Rossiter, S. J., Koch, J. L., \& Buggs, R. J. A. (2020). Convergent molecular evolution among ash species resistant to the emerald ash borer. Nature Ecology \& Evolution, 4, 1116-1128. https://doi.org/10.1038/s41559-020-1209-3

Knaus, B. J., \& Grünwald, N. J. (2017). VCFR: A package to manipulate and visualize variant call format data in R. Molecular Ecology Research, 17, 44-53.

Koch, J. L., Carey, D. W., Mason, M. E., Poland, T. M., \& Knight, K. S. (2015). Intraspecific variation in Fraxinus pennsylvanica responses to emerald ash borer (Agrilus planipennis). New Forests, 46, 9951011. https://doi.org/10.1007/s11056-015-9494-4

Kogan, M., \& Ortman, E. F. (1978). Antixenosis-a new term proposed to define Painter's "nonpreference" modality of resistance. Bulletin of the Entomological Society of America, 24, 175-176. https://doi. org/10.1093/besa/24.2.175

Kovacs, K. F., Mercader, R. J., Haight, R. G., Siegert, N. W., McCullough, D. G., \& Liebhold, A. M. (2011). The influence of satellite populations of emerald ash borer on projected economic costs in US communities, 2010-2020. Journal of Environmental Management, 92, 2170-2181. https://doi.org/10.1016/j.jenvm an.2011.03.043

Lelito, J. P., Böröczky, K., Jones, T. H., Fraser, I., Mastro, V. C., Tumlinson, J. H., \& Baker, T. C. (2009). Behavioral evidence for a contact sex pheromone component of the emerald ash borer, Agrilus planipennis Fairmarie. Journal of Chemical Ecology, 35, 104-110.

Lelito, J. P., Fraser, I., Mastro, V. C., Tumlinson, J. H., Böröczky, K., \& Baker, T. C. (2007). Visually mediated 'paratrooper copulations' in the mating behavior of Agrilus planipennis (Coleoptera: Buprestidae), a highly destructive invasive pest in North American ash trees. Journal of Insect Behavior, 20, 537-552.

Li, H., Handsaker, B., Wysoker, A., Fennell, T., Ruan, J., Homer, N., Marth, G., Abecasis, G., \& Durbin, R. (2009). The sequence alignment/map format and SAMtools. Bioinformatics, 25, 2078-2079. https://doi. org/10.1093/bioinformatics/btp352

Lischer, H. E. L., \& Excoffier, L. (2012). PGDSpider: An automated data conversion tool for connecting population genetics and genomics programs. Bioinformatics, 28, 298-299. https://doi.org/10.1093/ bioinformatics/btr642

Lotterhos, K. E., \& Whitlock, M. C. (2014). Evaluation of demographic history and neutral parameterization on the performance of $\mathrm{F}_{\mathrm{ST}}$ outlier tests. Molecular Ecology, 23, 2178-2192.

Marshall, J. M. (2020). Forest compositional changes after a decade of emerald ash borer. Forests, 11, 949. https://doi.org/10.3390/f1109 0949

Marshall, J. M., Porter, M. J., \& Storer, A. J. (2012). Predicting emerald ash borer, Agrilus planipennis (Coleoptera: Buprestidae), landing behavior on unwounded ash. Great Lakes Entomologist, 45, 29-39.

Marshall, J. M., Smith, E. L., Mech, R., \& Storer, A. J. (2013). Estimates of Agrilus planipennis infestation rates and potential survival of ash. American Midland Naturalist, 169, 179-193. https://doi. org/10.1674/0003-0031-169.1.179

Marshall, J. M., Storer, A. J., Fraser, I., Beachy, J. A., \& Mastro, V. C. (2009). Effectiveness of differing trap types for the detection of emerald ash borer (Coleoptera: Buprestidae). Environmental Entomology, 38, 1226-1234. https://doi.org/10.1603/022.038.0433

Marshall, J. M., Storer, A. J., Fraser, I., \& Mastro, V. C. (2010). Efficacy of trap and lure types for detection of Agrilus planipennis (Col., Buprestidae) at low density. Journal of Applied Entomology, 134, 296-302. 
Millers, I., Lachangce, D., Burkman, W. G., \& Allen, D. C. (1991). North American sugar maple decline project: organization and field methods. USDA Forest Service Gen. Tech. Rep. NE-154. Retrieved from http://www.fs.fed.us/ne/newtown_square/publications/techn ical_reports/pdfs/scanned/gtr154.pdf

Nielsen, R., Paul, J. S., Albrechtsen, A., \& Song, Y. S. (2011). Genotype and SNP calling from next-generation sequencing data. Nature Reviews Genetics, 12, 443. https://doi.org/10.1038/nrg2986

Painter, R. H. (1951). Insect Resistance in Crop Plants. The Macmillan Company.

Poland, T. M., \& McCullough, D. G. (2006). Emerald ash borer: Invasion of the urban forest and the threat to North America's ash resource. Journal of Forestry, 104, 118-124.

Purcell, S., Neale, B., Todd-Brown, K., Thomas, L., Ferraira, M. A. R., Bender, D., Maller, J., Sklar, P., de Bakker, P. I. W., Daly, M. J., \& Sham, P. C. (2007). PLINK: A tool set for whole-genome association and population-based linkage analysis. American Journal of Human Genetics, 81, 559-575.

Pureswaran, D. S., \& Poland, T. M. (2009). Host selection and feeding preference of Agrilus planipennis (Coleoptera: Buprestidae) on ash (Fraxinus spp.). Environmental Entomology, 38, 757-765.

R Core Team (2017). R: A language and environment for statistical computing. R Foundation for Statistical Computing. Retrieved from https:// www.R-project.org/

Rebek, E. J., Herms, D. A., \& Smitley, D. R. (2008). Interspecific variation in resistance to emerald ash borer (Coleoptera: Buprestidae) among North American and Asian ash (Fraxinus spp.). Environmental Entomologist, 37, 242-246.

Rigsby, C. M., Muilenburg, V., Tarpey, T., Herms, D. A., \& Cipollini, D. (2014). Oviposition preferences of Agrilus planipennis (Coleoptera: Buprestidae) for different ash species support the mother knows best hypothesis. Annals of the Entomological Society of America, 107, 773-781.

Russello, M. A., Waterhouse, M. D., Etter, P. D., \& Johnson, E. A. (2015). From promise to practice: Pairing non-invasive sampling with genomics in conservation. PeerJ, 3, e1106. https://doi.org/10.7717/ peerj.1106

Schlesinger, R. C. (1990). Fraxinus americana L. white ash. In R. M. Burns \& B. H. Honkala (Eds.), Silvics of North America. Volume 2: Hardwoods. Agriculture Handbook 654. US Department of Agriculture, US Forest Service.

Sessa, G., D'Ascenzo, M., \& Martin, G. B. (2000). The major site of the Pti1 kinase phosphorylated by the Pto kinase is located in the activation domain and is required for Pto-Pti1 physical interaction. FEBS Journal, 267, 171-178.

Siegert, N. W., McCullough, D. G., Liebhold, A. M., \& Telewski, F. W. (2014). Dendrochronological reconstruction of the epicenter and early spread of emerald ash borer in North America. Diversity and Distributions, 20, 847-858.

Sousa, V., \& Hey, J. (2013). Understanding the origin of species with genome-scale data: Modelling gene flow. Nature Reviews Genetics, 14, 404-414. https://doi.org/10.1038/nrg3446

Tanis, S. R., \& McCullough, D. G. (2012). Differential persistence of blue ash and white ash following emerald ash borer invasion. Canadian Journal of Forest Research, 42, 1542-1550. https://doi.org/10.1139/ x2012-103

Villari, C., Herms, D. A., Whitehill, J. G., Cipollini, D., \& Bonello, P. (2016). Progress and gaps in understanding mechanisms of ash tree resistance to emerald ash borer, a model for wood-boring insects that kill angiosperms. New Phytologist, 209, 63-79. https://doi. org/10.1111/nph.13604

Wallander, E. (2008). Systematics of Fraxinus (Oleaceae) and evolution of dioecy. Plant Systematics and Evolution, 273, 25-49. https://doi. org/10.1007/s00606-008-0005-3

Wei, X., Wu, Y., Reardon, R., Sun, T. H., Lu, M., \& Sun, J. H. (2007). Biology and damage traits of emerald ash borer (Agrilus planipennis Fairmaire) in China. Insect Science, 14, 367-373. https://doi. org/10.1111/j.1744-7917.2007.00163.x

Whitehill, J. G., Opiyo, S. O., Koch, J. L., Herms, D. A., Cipollini, D. F., \& Bonello, P. (2012). Interspecific comparison of constitutive ash phloem phenolic chemistry reveals compounds unique to Manchurian ash, a species resistant to emerald ash borer. Journal of Chemical Ecology, 38, 499-511. https://doi.org/10.1007/s1088 6-012-0125-7

Whitehill, J. G., Popova-Butler, A., Green-Church, K. B., Koch, J. L., Herms, D. A., \& Bonello, P. (2011). Interspecific proteomic comparisons reveal ash phloem genes potentially involved in constitutive resistance to the emerald ash borer. PLoS One, 6, e24863. https:// doi.org/10.1371/journal.pone.0024863

Whittemore, A. T., Campbell, J. J. N., Xia, Z.-L., Carlson, C. H., Atha, D., \& Olsen, R. T. (2018). Ploidy variation in Fraxinus L. (Oleaceae) of Eastern North America: Genome size diversity and taxonomy in a suddenly endangered genus. International Journal of Plant Sciences, 179, 377-389.

How to cite this article: Hale, C. E., Jordan, M. A., Iriarte, G. Broders, K., Storer, A. J., Nalam, V. J., \& Marshall, J. M. (2021). Genome-wide SNP identification in Fraxinus linking genetic characteristics to tolerance of Agrilus planipennis. Ecology and Evolution, 11, 14775-14788. https://doi.org/10.1002/ ece3.8163 